\title{
A Case of Pseudomyxoma Peritonei With a Synchronous Inguinal Hernia
}

\author{
Stephanie Yee ${ }^{\mathrm{a}}$, Tyrell Daniel ${ }^{\mathrm{a}}$, Ahmad Hlayhel ${ }^{\mathrm{a}, \mathrm{b}}$, Lindsey Foran ${ }^{\mathrm{a}}$, \\ Jamshed Zuberia ${ }^{\mathrm{a}}$, Mark Ingram ${ }^{\mathrm{a}}$
}

\begin{abstract}
Pseudomyxoma peritonei (PMP) is a rare disease that most commonly results from the rupture of an appendiceal mucinous neoplasm. It is characterized by mucinous ascites that disseminates throughout the abdomen and pelvis, and at times to the inguinal canal, in which some patients may complain of inguinal hernia as the initial complaint. The incidence of PMP with concomitant inguinal hernia is reported to be $7.3-9.6 \%$ in the current literature. We report a 70-year-old Hispanic male who initially presented with signs of a right inguinal hernia, and on computed tomography (CT) scan, was subsequently found to have a large collection of right lower quadrant cysts extending into the right inguinal canal and hemiscrotum. Patient underwent exploratory laparotomy and was found to have a large right retroperitoneal mass with PMP with mucinous contents herniating through the right inguinal canal encased within peritoneal sac and had surgical debulking of peritoneal cavity and right hemiscrotum. The patient was discharged 9 days later. PMP that presents with an inguinal hernia is a special entity that warrants a discussion on the optimal management of inguinal involvement of a mucinous tumor.
\end{abstract}

Keywords: Pseudomyxoma peritonei; Surgical debulking; Inguinal hernia

\section{Introduction}

Pseudomyxoma peritonei (PMP) is a rare malignant disease with an estimated annual incidence of one to two cases per million persons per year [1]. PMP is defined by the accumulation of mucin secondary to the rupture of a mucinous neoplasm which arises in the appendix in about $90 \%$ of cases [1]. The remaining $10 \%$ of cases of PMP can originate from an extra-

Manuscript submitted June 22, 2020, accepted July 25, 2020

Published online August 22, 2020

aDepartment of Surgery, St. Joseph's University Medical Center, Paterson, NJ 07503, USA

bCorresponding Author: Ahmad Hlayhel, Department of Surgery, St. Joseph's University Medical Center, 703 Main St., Paterson, NJ 07503, USA.

Email: afhlayhel@gmail.com

doi: https://doi.org/10.14740/jcs415 appendicular source such as the colon, ovary, small bowel, urachus, or pancreas [2-6]. The mucin producing tumor cells accumulate and become widely spread within the peritoneal cavity. These cells can invade the omentum or other viscera, eventually leading to massive abdominal distension and possibly mechanical or functional bowel dysfunction [7]. It is not uncommon for the mucinous fluid to extend into the inguinal canal, causing an inguinal or scrotal bulge. The standard of care of PMP, with or without the presence of an inguinal hernia, remains the combination of complete cytoreductive surgery (CRS) with hyperthermic intraperitoneal chemotherapy (HIPEC) $[8,9]$. We present a case of a patient with PMP presenting as an inguinal hernia who underwent surgical debulking without HIPEC.

\section{Case Report}

A 70-year-old Hispanic male with a history of obesity, hypertension and retroperitoneal mass presented to the emergency department (ED) with chronic abdominal pain which was worse in the right lower quadrant. Two months prior to presentation, the patient was seen in the surgical clinic for evaluation of a right inguinal hernia. Given that the patient reported a history of a retroperitoneal mass which we did not have records of, a computed tomography (CT) of abdomen and pelvis for pre-operative planning was obtained. The CT scan at that time was notable for multiple bilateral renal cysts, with the largest right renal cyst measuring approximately $38 \mathrm{~cm}$ with its most inferior portion extending to the scrotum. The patient was lost to follow-up and now presented to the ED with abdominal pain. The patient reported a 1-year history of a right inguinal hernia and chronic right sided abdominal pain. $\mathrm{He}$ also reported decreased appetite and nausea with per os (PO) intake associated with intermittent diarrhea, and denied vomiting, melena, and hematochezia. Vital signs on admission were within normal limits. On physical examination, the abdomen was noted to be soft, non-distended with mild right upper and lower quadrant tenderness without peritoneal signs. His exam was also notable for an enlarged right scrotum with non-reducible palpable mass without overlying erythema. A repeat $\mathrm{CT}$ of abdomen and pelvis scan demonstrated a persistent right retroperitoneal mass with the intraperitoneal portion measuring approximately $12.5 \times 17.5 \times 23 \mathrm{~cm}$ but now with a thickened wall, change in contour, and air inside the cystic mass that raised concern for a possible fistulous tract with the bowel 


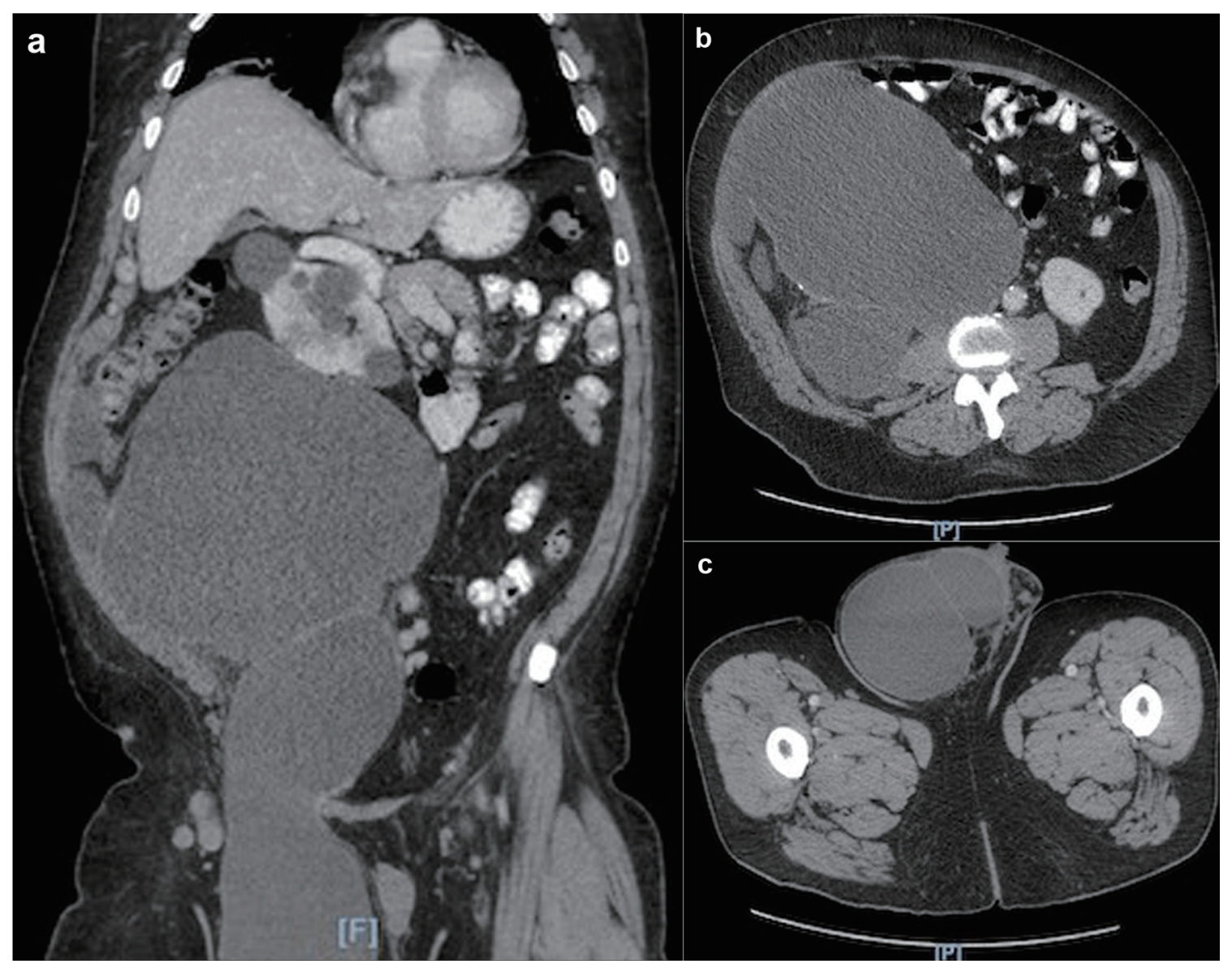

Figure 1. (a) CT scan showing large right retroperitoneal mass extending into the right scrotum. (b) CT scan axial view showing large right retroperitoneal mass. (c) CT scan axial view showing the extension of the retroperitoneal mass into the right scrotum. CT: computed tomography.

(Fig. 1). CT scan was also notable for a lipomatous appearing gastric mass. On day 2 in hospital, patient reported dark stools and required transfusion of two units packed red blood cells. Esophagogastroduodenoscopy (EGD) was performed on day 2 in hospital to evaluate the gastric mass and was notable for coffee ground material in the stomach with two oozing cratered duodenal ulcers with a visible vessel in part I of duodenum. The area was successfully treated with epinephrine injection along with electrocautery coagulation. A gastric lipoma was visualized and biopsied. Pathology report confirmed a gastric lipoma, chronic gastritis and focal staining of Helicobacter pylori (H. pylori). His case was discussed interdepartmentally, and the decision was made to take the patient to the operating room (OR) on this admission so as to not lose the patient to follow-up once again. As such, on day 9 in hospital, patient was taken to the OR for a planned exploratory laparotomy with pre-operative right ureteral stent placement. Immediately upon entry of the peritoneal cavity, there was mucinous fluid throughout the peritoneal cavity and there was a notably large right retroperitoneal mass adherent to the adjacent omentum and small bowel upon initial inspection (Fig. 2a). An omentectomy and resection of $4 \mathrm{~cm}$ of involved distal ileum was performed (Fig. 2b). The right ureter that was displaced to the mid abdomen was isolated and mobilized. Next an attempt to resect the retroperitoneal mass with gross debulking was performed. The posterior peritoneal lining was difficult to dissect and was left behind. The cystic mass tracked in through the right inguinal canal and the peritoneal lining was debulked. A Penrose drain was left in the right hemiscrotum. Given the extent of residual macroscopic disease, HIPEC was not deemed an option for this patient. Surgical pathology of the sampled umbilical nodule (Fig. 3), peritoneal tissue and retroperitoneal mass (Fig. 4) was notable for extensive fibroadipose tissue with extensive dissecting mucin consistent with PMP, a low-grade mucinous neoplasm most consistent with an appendiceal primary source.

\section{Discussion}

PMP is a rare disease that arises from the appendix in $90 \%$ of cases, and rarely from extra-appendiceal sources [2-6]. Early diagnosis of this neoplasm may show limited extension of disease, but in $30 \%$ of cases the mucin accumulation is seen extending throughout the abdomen leading to symptoms [10]. Although PMP has a very low risk of systemic dissemination, there is still concern of symptoms that arise from its diffuse and 


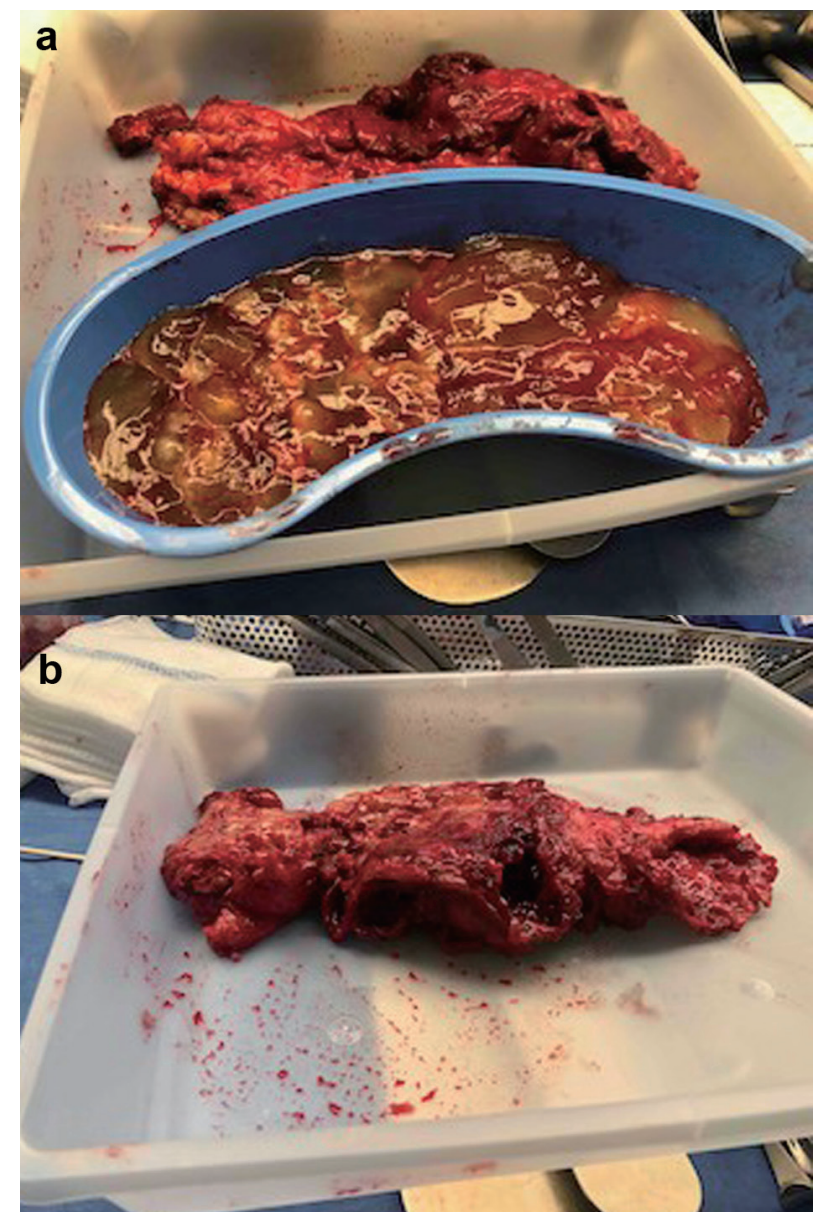

Figure 2. (a) Intraoperative photo of the mucinous ascites seen upon initial entry into peritoneal cavity. (b) Intraoperative photo of resected distal ileum densely adhered to cystic material.

slow spread in the peritoneal cavity $[11,12]$. These symptoms include abdominal distension, pain and bowel dysfunction [10]. Some complications that may occur due to the potentially massive disease burden of PMP are mass effect, malnutrition,

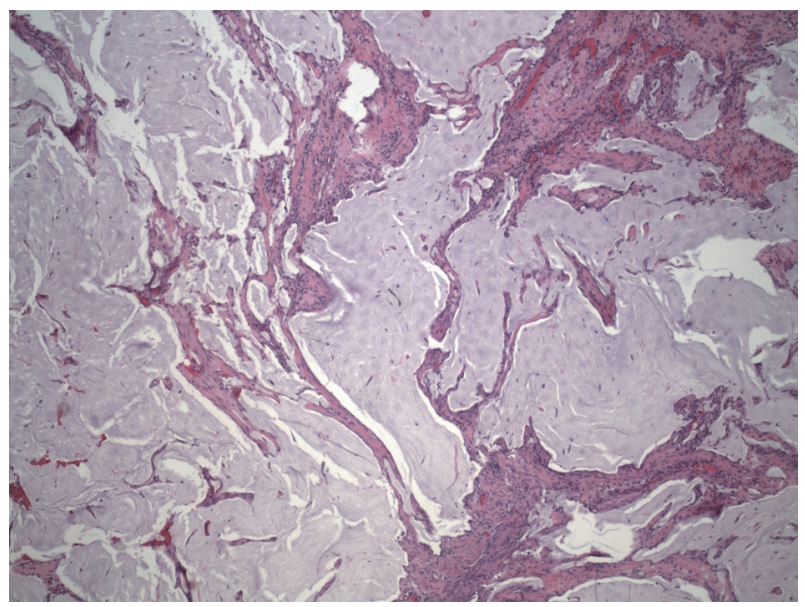

Figure 3. Umbilical nodule showing dissecting mucin.

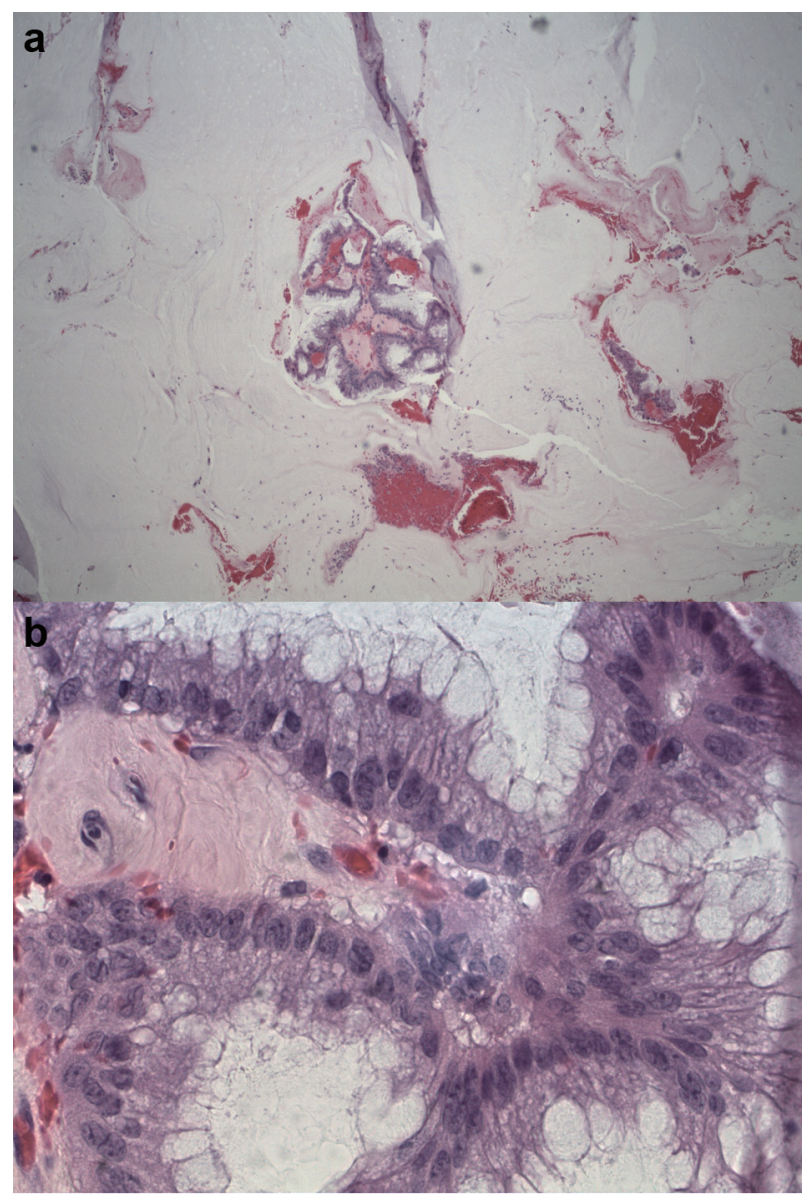

Figure 4. (a) Low-power view of mass showing abundant mucin and a few fragments of neoplastic mucinous glandular epithelium floating within the mucin. (b) High-power view of mass showing abundant mucin and a few fragments of neoplastic mucinous glandular epithelium floating within the mucin.

bowel obstruction and death without treatment [12]. Hence, an early diagnosis and intervention is essential to improving the outcomes of patients with PMP.

Effective diagnosis of PMP is necessary to treat the disease early before complications arise. Current imaging modalities available to assess for PMP include CT, positron emission tomography (PET)-CT and magnetic resonance imaging (MRI) but these are limited in their ability to detect low volume disease recurrence. CT remains the recommended modality due to its ease of interpretation, affordability and availability [12]. However, Chua et al demonstrated that the use of CT to highlight the degree of peritoneal carcinomatosis evaluated by the peritoneal cancer index (PCI) constantly underestimated the spread of PMP in the peritoneum [13]. Hence, MRI may be necessary for diagnosing and monitoring patients with known PMP due to its fast imaging sequences with high contrast resolution [14]. Definitive diagnosis of PMP is made intraoperatively along with histopathology of lesions obtained as surgical specimens.

Treatment modalities typically entail surgical debulking, chemotherapy or a combination of both. The gold standard of 
treatment for PMP continues to be CRS and HIPEC. CRS includes peritonectomy procedures with any necessary visceral resections, while HIPEC involves warmed chemotherapy solution that is infused and circulated in the peritoneal cavity [15]. The benefits of CRS and HIPEC on overall survival and disease-free survival in patients with peritoneal metastases may be predicted via the extent of peritoneal involvement evaluated by the PCI and the completeness of the CRS [16]. Sometimes CRS may not be possible due to high tumor burden that may require resecting organs that may impair patients' quality of life and increase postoperative morbidity [12]. Surgical debulking by itself has been found to be beneficial for huge tumor burden as symptoms gradually decreased over a period of 23 months and $50 \%$ of patients had no related symptoms at median follow-up of 24.5 months [12].

While current literature does provide general guidelines for the diagnosis and management of PMP, there are no clear recommendations for the management of an inguinal defect in the setting of PMP. Current literature reports a 7.3-9.6\% incidence of PMP diagnosed along with an inguinal hernia [1, 17]. A study by Sugarbaker in 2016 found that males were five times more likely to develop inguinal hernias with PMP than females [17]. Sugarbaker reviewed the outcomes of 17 patients with PMP who had synchronous or metachronous inguinal hernia [17]. All 17 patients underwent peritonectomy, complete extraction of the peritoneal lining of the inguinal canal to lowest possible level. Drains were placed for HIPEC mitomycin $\mathrm{C}$, doxorubicin, 5-fluorouracil and leucovorin for 90 min at $42{ }^{\circ} \mathrm{C}$ with care to repeatedly lavage the open inguinal canal as well. After the HIPEC procedure, the deperitonealized inguinal canal was left open to eventually close by fibrosis. Of note, 10 patients had inguinal hernia repair prior to definitive treatment of PMP while seven patients had hernia repair synchronously with treatment of PMP. At 3 months to 8 years follow-up, none of these patients had recurrence of hernia or required reoperations for PMP recurrence in the inguinal region. This is the only study that proposes a treatment strategy specifically for synchronous or metachronous inguinal hernia in patients with PMP.

Another aspect to consider in the treatment of PMP with inguinal involvement is the route of the HIPEC therapy. A retrospective review of 5 patients with PMP with inguinal involvement found that CRS with HIPEC infusion to include perfusion through the groin may be considered safe and effective, where 4 patients were alive and disease free at mean follow-up of 27 months [18]. One patient suffered retroperitoneal recurrence and died at 7 months due to chemotherapy related toxicity. This study demonstrates the need for further investigation of a modified HIPEC technique to target the involved inguinal canal.

\section{Conclusions}

Inguinal involvement in patients with PMP is not uncommon and its management and outcomes warrant further investigation. Patients who initially present with an inguinal hernia who may present with an underlying PMP should be carefully identified and worked up with a CT scan; however, risk factors for suspecting PMP are yet to be defined. Once a patient is suspected to have PMP, groin involvement should be considered in the surgical planning. Inguinal canal involvement in PMP may benefit from complete deperitonealization of the inguinal canal for the goal of complete tumor removal or HIPEC through the inguinal canal as reported in some studies. Further investigation on the management and outcomes of PMP specifically with inguinal involvement is needed.

\section{Acknowledgments}

The authors would like to thank St. Joseph's University Medical Center for the support.

\section{Financial Disclosure}

This research did not receive any specific grant from funding agencies in the public, commercial, or not-for-profit sectors.

\section{Conflict of Interest}

None to declare.

\section{Informed Consent}

Not applicable.

\section{Author Contributions}

SY, TD, AH, and LF drafted the manuscript. JZ and MI did the critical revision.

\section{Data Availability}

The authors declare that data supporting the findings of this study are available within the article.

\section{References}

1. Esquivel J, Sugarbaker PH. Clinical presentation of the pseudomyxoma peritonei syndrome. Br J Surg. 2000;87(10):1414-1418.

2. Ronnett BM, Seidman JD. Mucinous tumors arising in ovarian mature cystic teratomas: relationship to the clinical syndrome of pseudomyxoma peritonei. Am J Surg Pathol. 2003;27(5):650-657.

3. Carr NJ, Finch J, Ilesley IC, Chandrakumaran K, Mohamed F, Mirnezami A, Cecil T, et al. Pathology and prognosis in pseudomyxoma peritonei: a review of 274 cases. J Clin Pathol. 2012;65(10):919-923.

4. Lemahieu J, D'Hoore A, Deloose S, Sciot R, Moerman P. 
Pseudomyxoma peritonei originating from an intestinal duplication. Case Rep Pathol. 2013;2013:608016.

5. Liu Y, Ishibashi H, Hirano M, Takeshita K, Mizumoto A, Ichinose M, Nishino E, et al. Cytoreductive surgery plus hyperthermic intraperitoneal chemotherapy for pseudomyxoma peritonei arising from urachus. Ann Surg Oncol. 2015;22(8):2799-2805.

6. Rosenberger LH, Stein LH, Witkiewicz AK, Kennedy EP, Yeo CJ. Intraductal papillary mucinous neoplasm (IPMN) with extra-pancreatic mucin: a case series and review of the literature. J Gastrointest Surg. 2012;16(4):762-770.

7. Miner TJ, Shia J, Jaques DP, Klimstra DS, Brennan MF, Coit DG. Long-term survival following treatment of pseudomyxoma peritonei: an analysis of surgical therapy. Ann Surg. 2005;241(2):300-308.

8. Moran B, Baratti D, Yan TD, Kusamura S, Deraco M. Consensus statement on the loco-regional treatment of appendiceal mucinous neoplasms with peritoneal dissemination (pseudomyxoma peritonei). J Surg Oncol. 2008;98(4):277-282.

9. Deraco M, Baratti D, Inglese MG, Allaria B, Andreola S, Gavazzi C, Kusamura S. Peritonectomy and intraperitoneal hyperthermic perfusion (IPHP): a strategy that has confirmed its efficacy in patients with pseudomyxoma peritonei. Ann Surg Oncol. 2004;11(4):393-398.

10. Galan A, Rousset P, Mercier F, Kepenekian V, Valette PJ, Glehen O, Passot G. Overall survival of pseudomyxoma peritonei and peritoneal mesothelioma patients after cytoreductive surgery and hyperthermic intraperitoneal chemotherapy can be predicted by computed tomography quantified sarcopenia. Eur J Surg Oncol. 2018;44(11):1818-1823.

11. Darr U, Renno A, Alkully T, Khan Z, Tiwari A, Zeb W,
Purdy J, et al. Diagnosis of Pseudomyxoma peritonei via endoscopic ultrasound guided fine needle aspiration: a case report and review of literature. Scand J Gastroenterol. 2017;52(5):609-612.

12. Delhorme JB, Elias D, Varatharajah S, Benhaim L, Dumont F, Honore C, Goere D. Can a benefit be expected from surgical debulking of unresectable pseudomyxoma peritonei? Ann Surg Oncol. 2016;23(5):1618-1624.

13. Chua TC, Al-Zahrani A, Saxena A, Glenn D, Liauw W, Zhao J, Morris DL. Determining the association between preoperative computed tomography findings and postoperative outcomes after cytoreductive surgery and perioperative intraperitoneal chemotherapy for pseudomyxoma peritonei. Ann Surg Oncol. 2011;18(6):1582-1589.

14. Low RN. MR imaging of the peritoneal spread of malignancy. Abdom Imaging. 2007;32(3):267-283.

15. Sugarbaker PH. New standard of care for appendiceal epithelial neoplasms and pseudomyxoma peritonei syndrome? Lancet Oncol. 2006;7(1):69-76.

16. Chua TC, Yan TD, Saxena A, Morris DL. Should the treatment of peritoneal carcinomatosis by cytoreductive surgery and hyperthermic intraperitoneal chemotherapy still be regarded as a highly morbid procedure? A systematic review of morbidity and mortality. Ann Surg. 2009;249(6):900-907.

17. Sugarbaker PH. Management of an inguinal hernia in patients with pseudomyxoma peritonei. Eur J Surg Oncol. 2017;43(6):1083-1087.

18. Shachar Y, Adileh M, Keidar A, Eid L, Hubert A, Temper M, Azam S, et al. Management of inguinal involvement of peritoneal surface malignancies by cytoreduction and HIPEC with inguinal perfusion. J Cancer. 2015;6(3):243246. 